\title{
碳点修饰多孔ZnO纳米棒增强光催化性能
}

\author{
冯 昌 ${ }^{1}$ 邓晓燕 ${ }^{1, *}$ 倪晓晓 ${ }^{2}$ 李卫兵 ${ }^{1}$ \\ ( '青岛科技大学环境与安全工程学院, 山东青岛 266042; 2 2 青岛市城市排水监测站, 山东青岛 266002)
}

\begin{abstract}
摘要: 首先采用溶剂热法制备具有良好光催化降解性能的多孔ZnO纳米棒, 随后采用化学沉积法在ZnO纳米 棒表面修饰碳点, 成功制备碳点修饰多孔ZnO纳米棒系列光催化剂. 通过X射线衍射(XRD)、扫描电子显微镜 (SEM)、透射电子显微镜(TEM)、X射线光电子能谱(XPS)、紫外-可见(UV-Vis)分光光谱等技术对系列样品 进行表征. 通过测试系列样品在氙灯光源照射下, 降解有机污染物(罗丹明 $B$ 和苯酚)的光催化性能, 发现碳点修 饰多孔ZnO纳米棒能有效增强ZnO的光催化性能, 其中质量分数为 $1.2 \%$ 碳点修饰 ZnO纳米棒 $(\mathrm{CZn} 1.2)$ 表现出 最高的光催化降解罗丹明 $\mathrm{B}$ 活性, 为纯多孔ZnO纳米棒的 2.5 倍; 同时, 在氙灯光源照射下, 碳点修饰多孔ZnO纳 米棒具有很强的氧化性, 能够催化苯环的开环反应, 实现对苯酚的降解. 其光催化活性的增强在于碳点修饰多 孔ZnO光催化材料中, 碳点作为光生电子受体, 促进半导体光生电子与空穴的有效分离, 从而增强光催化性能.
\end{abstract}

关键词: 半导体光催化; $\mathrm{ZnO}$; 碳点; 降解有机污染物; 修饰光催化剂

中图分类号: 0643

\section{Fabrication of Carbon Dots Modified Porous ZnO Nanorods with Enhanced Photocatalytic Activity}

\author{
FENG Chang $^{1} \quad$ DENG Xiao-Yan ${ }^{1, *} \quad$ NI Xiao-Xiao ${ }^{2} \quad$ LI Wei-Bing $^{1}$ \\ ( ${ }^{1}$ School of Environment and Safety Engineering, Qingdao University of Science and Technology, Qingdao 266042, Shandong Province, \\ P. R. China; ${ }^{2}$ Qingdao Municipal Drainage Monitoring Center, Qingdao 266002, Shandong Province, P. R. China)
}

\begin{abstract}
Porous $\mathrm{ZnO}$ nanorods that displayed excellent photocatalytic degradation of organic pollutants (RhB and phenol) were prepared via a solvent thermal method followed by surface modification with carbon dots (C-dots) using a deposition method. The photocatalysts were characterized using X-ray diffraction (XRD), scanning electron microscopy (SEM), transmission electron microscopy (TEM), X-ray photoelectron spectroscopy (XPS), and ultraviolet-visible (UV-Vis) spectroscopy. The degradation of the organic pollutants using the nanorods was tested under Xe-light illumination and was enhanced following C-dot modification. Nanorods that were modified by the $\mathrm{C}$-dots at a mass fraction of $1.2 \%$ (CZn1.2) exhibited the highest photocatalytic activity for the degradation of $\mathrm{RhB}$, which was 2.5 times of the pure porous $\mathrm{ZnO}$ nanorods. Additionally, the modified nanorods with strangely oxidation ability could catalyze the degradation of phenol by open-rings reaction under Xe-light illumination. The improved photocatalytic activity was attributed to the effective separation of the photogenerated electrons and holes, in which the C-dots served as the receptor of the photogenerated electrons.
\end{abstract}

Key Words: Semiconductor photocatalysis; ZnO; C-dots; Degradation of organic pollutants; Modified photocatalyst

\footnotetext{
Received: August 7, 2015; Revised: October 27, 2015; Published on Web: October 28, 2015.

"Corresponding author. Email: deng_xiao_yan@126.com; Tel: +86-18562577395.

The project was supported by the National Natural Science Foundation of China (41376126) and Foundation of Key Laboratory of Marine Environmental Corrosion and Bio-fouling, Institute of Oceanology, Chinese Academy of Sciences.

国家自然科学基金(41376126)及中国科学院海洋研究所海洋环境腐蚀与生物污损重点实验室开放基金资助项目
}

(C) Editorial office of Acta Physico-Chimica Sinica 


\section{1 引 言}

随着科学技术飞速发展, 环境污染和能源短缺 问题日益突出, 而光催化技术被认为是解决这两大 问题的最有效手段. ${ }^{1-5} \mathrm{ZnO}$ 半导体光催化剂因为廉 价、无污染、易制备等特点被广泛应用于光催化 领域..$^{6-8}$ 然而 $\mathrm{ZnO}$ 本身因禁带宽度(常温下 $E_{\mathrm{g}}=3.37$ $e V)$ 较宽, 只能吸收太阳光中的紫外光部分, 致使 $\mathrm{ZnO}$ 本身的光催化效率较低. ${ }^{9,10}$ 如何提高 $\mathrm{ZnO}$ 的光 催化性能成为光催化研究领域的热点, Zhu等 ${ }^{11}$ 采用 溶剂热法制备了 $\mathrm{ZnO}$ 空心球, 并研究其优异的光催 化性能, 得出空心结构可以捕获大量紫外光子增强 光催化降解能力的结论. Samadi等 ${ }^{12}$ 制备了 C掺杂的 $\mathrm{ZnO}$ 光催化剂, 并研究 $\mathrm{ZnO}$ 的结构及光催化性能, 发 现在 $\mathrm{ZnO}$ 中形成了 $\mathrm{Zn}-\mathrm{O}-\mathrm{C}$ 键, 减小了 $\mathrm{ZnO}$ 的晶粒 尺寸, 从而抑制光生电子-空穴的复合率, 使 $\mathrm{ZnO}$ 的 禁带宽度从 $3.11 \mathrm{eV}$ 降至 $2.94 \mathrm{eV}$, 从而增强了对可见 光的吸收, 提高了 $\mathrm{ZnO}$ 光催化活性. $\mathrm{Bu}$ 和 $\mathrm{Chen}^{13}$ 利用 $\mathrm{CdS}$ 敏化氢处理 $\mathrm{ZnO}$ 纳米棒阵列来研究其光电化学 性能, 发现其良好的光解水制氢性能, 研究表明, $\mathrm{CdS}$ 敏化氢处理 $\mathrm{ZnO}$ 纳米棒阵列光电化学性能明显 优于纯 $\mathrm{ZnO}$ 纳米棒阵列. 经过分析, 发现通过 $\mathrm{CdS}$ 的 敏化能有效提高载流子浓度和电子迁移率, 使光生 电子寿命延长, 具有良好的制氢性能. 因此, 通过不 同途径如控制形貌、掺杂、包覆等手段改性 $\mathrm{ZnO}$ 半 导体材料可以有效利用光能, 提高光生电子与空穴 的分离效率, 提高其光催化活性. ${ }^{14-17}$

碳点 $(\mathrm{C}-\mathrm{dots})$ 作为一种尺寸在 $10 \mathrm{~nm}$ 以下的新型 碳纳米材料, 因其优良的光电性质被广泛应用于光 催化领域. ${ }^{18,19} \mathrm{Li}$ 等 ${ }^{20}$ 合成碳点@ $\mathrm{TiO}_{2}$ 复合材料进行有 机染料的光催化降解测试, 发现碳点首先吸收太阳 光能, 然后通过上转换机制发射出325-425 nm的短 波, 进一步激发 $\mathrm{TiO}_{2}$ 产生光生电子空穴对从而完成 对染料的降解. Ming 等 ${ }^{21}$ 通过电化学法制备了碳点, 表现出类过氧化物酶的作用, 进一步修饰 $\mathrm{TiO}_{2}$, 发现 $\mathrm{TiO}_{2} / \mathrm{C}$-dots光催化剂具有良好的可见光催化活性. $\mathrm{De}$ 等 ${ }^{22}$ 制备碳点还原纳米杂化 $\mathrm{Cu}_{2} \mathrm{O}$ 与超支化环氧树 脂复合材料, 并研究其机械性能、热能与光催化性 能. 碳点在修饰光催化材料中主要作为上转换机制 的主体和光生电子转移体, 能够拓展光催化材料的 吸光范围, 提高光生电子与空穴的分离效率, 增强 光催化性能. ${ }^{23-25}$

$\mathrm{TiO}_{2} / \mathrm{C}$-dots 复合光催化剂能够明显提升纯
$\mathrm{TiO}_{2}$ 的光催化性能, ${ }^{20,21} \mathrm{ZnO}$ 作为优良的光催化剂, 其 电子迁移率为 $\mathrm{TiO}_{2}$ 的数十倍, ${ }^{26}$ 并且有较高的晶体质 量和良好的化学稳定性, 被广泛应用于光催化和光 电化学领域. ${ }^{27,28}$ 本文通过电化学法制备碳点并修饰 多孔ZnO纳米棒, 成功合成系列碳点修饰 $\mathrm{ZnO}$ 样品, 并对其进行物理表征, 研究其光催化降解有机污染 物的性能.

\section{2 实验部分}

本实验所有试剂药品均来自国药集团化学试 剂有限公司, 纯度为分析纯, 实验过程中未经进一 步处理.

\section{1 碳点的电化学方法合成}

碳点通过典型的电化学方法合成, ${ }^{21,29}$ 利用恒电 位仪将两根石墨棒( $99.99 \%$, Alfa Aesar Co. Ltd.)作 为阴阳极放入超纯水中, 在两电极之间施加 $30 \mathrm{~V}$ 的 直流恒电压, 缓慢摚拌 $120 \mathrm{~h}$ 后, 以10000 r· $\mathrm{min}^{-1}$ 转速 离心去除沉淀, 将余下棕黄色溶液 $80{ }^{\circ} \mathrm{C}$ 进行干燥, 称重配制 $400 \mathrm{mg} \cdot \mathrm{L}^{-1}$ 碳点溶液, 之后取 $30 \mathrm{~mL}$ 碳点溶 液与 $30 \mathrm{~mL}$ 氨水 $(25 \%-28 \%(w))$ 混合倒入 $100 \mathrm{~mL}$ 聚四 氟乙烯内祄的反应釜于 $150{ }^{\circ} \mathrm{C} 反$ 应 $5 \mathrm{~h}$, 之后鼓风通 入空气于室温下晾至 $\mathrm{pH}$ 值约为 7 .

\section{2 溶剂热法合成多孔ZnO纳米棒材料}

合成多孔ZnO纳米棒主要参照Peng等 ${ }^{30}$ 报道, 并 对合成过程做了进一步的优化. 分别配制含有 10 $\mathrm{mmol} \mathrm{Zn}\left(\mathrm{NO}_{3}\right)_{2} \cdot 6 \mathrm{H}_{2} \mathrm{O}$ 和 $10 \mathrm{mmol} \mathrm{H}_{2} \mathrm{C}_{2} \mathrm{O}_{4} \cdot 2 \mathrm{H}_{2} \mathrm{O}$ 的无 水乙醇溶液各 $20 \mathrm{~mL}$, 并搅拌使其完全溶解. 搅拌状 态下, 将含有草酸的混合液迅速倒入 $\mathrm{Zn}\left(\mathrm{NO}_{3}\right)_{2}$ 混合 液中混合形成匀胶前体, 将混合前体倒入聚四氟乙 烯内祄的高压釜 $(50 \mathrm{~mL})$ 中. $130^{\circ} \mathrm{C} 反$ 应 $10 \mathrm{~h}$, 取出, 冷至室温, 离心收集, 并用去离子水洗三遍, 无水乙 醇洗两遍, 离心后 $80{ }^{\circ} \mathrm{C}$ 干燥 $24 \mathrm{~h}$ 得到 $\mathrm{ZnC}_{2} \mathrm{O}_{4}$. 然后, 所得产物 $420^{\circ} \mathrm{C}$ 煅烧 $2 \mathrm{~h}$, 得到纯相多孔ZnO纳米棒 ( $\mathrm{ZnO} \mathrm{NR})$.

\section{3 合成碳点修饰多孔ZnO纳米棒系列光催化 材料}

取一定量多孔ZnO纳米棒于 $50 \mathrm{~mL}$ 蒸馏水中超 声 $10 \mathrm{~min}$, 分别滴加质量分数为 $0.2 \% 、 0.4 \%$ 、 $0.8 \% 、 1.2 \% 、 1.6 \% 、 2.0 \%$ 的碳点于 $\mathrm{ZnO}$ 混合液中 搅拌 $4 \mathrm{~h}$, 之后 $8000 \mathrm{r} \cdot \mathrm{min}^{-1}$ 离心, 水洗离心, $100{ }^{\circ} \mathrm{C}$ 干 燥 $12 \mathrm{~h}$, 并标记为 $\mathrm{CZn} 0.2 、 \mathrm{CZn} 0.4 、 \mathrm{CZn} 0.8$ 、 CZn1.2、CZn1.6、CZn2.0. 


\section{4 系列样品物理表征}

通过D/MAX-2500/PC X射线衍射分析仪 (XRD, 日本理学)表征了碳点修饰 $\mathrm{ZnO}$ 纳米棒系列样品的 晶相组成; 通过JSM-6700F场发射电子扫描显微镜 (FE-SEM, 日本电子)观察制备样品的表面微观形貌; 通过JEM-2100F透射电子显微镜(TEM, 日本电子) 观察了样品的多孔棒状结构、颗粒之间的结合以 及修饰碳点后 $\mathrm{ZnO}$ 颗粒表面变化; 通过 Thermo ESCALAB 250XI X射线光电子能谱(XPS, 英国赛 默飞世尔科技)分析系列样品元素组成及化合态; TU-1901紫外-可见漫反射光谱仪(UV-Vis DRS, 普 析通用)表征系列样品的光学吸收性能.

\section{5 系列样品光催化性能测试}

光催化降解有机污染物实验中光催化剂材料 使用量为 $0.1 \mathrm{~g}$, 降解的有机染料为 $100 \mathrm{~mL}$ 浓度为 10 $\mathrm{mg} \cdot \mathrm{L}^{-1}$ 的罗丹明 $\mathrm{B}(\mathrm{RhB})$ 溶液. 降解的难降解有机物 为 $100 \mathrm{~mL}$ 浓度为 $20 \mathrm{mg} \cdot \mathrm{L}^{-1}$ 的苯酚溶液. 光催化降解 实验开始前将有机污染物溶液和系列光催化剂材 料混合并在暗态条件下搅拌 $30 \mathrm{~min}$ 达到吸附平衡. 使用外照式 $300 \mathrm{~W}$ 氙灯光源(PLS-SXE300, 北京泊 菲莱科技有限公司), 氙灯光照射光照强度为 750 $\mathrm{mW} \cdot \mathrm{cm}^{-2}$, 在氙灯光源上添加滤波片 $(\geq 420 \mathrm{~nm})$ 模 拟可见光测试可见光降解有机染料的性能(光照强 度为 $\left.550 \mathrm{~mW} \cdot \mathrm{cm}^{-2}\right)$. 光源与有机污染物液面距离为 $10 \mathrm{~cm}$, 通过 $25^{\circ} \mathrm{C}$ 的冷凝水系统维持降解时温度的 恒定. 降解过程中采用 $8000 \mathrm{r} \cdot \mathrm{min}^{-1}$ 的转速离心取上 清液进行吸光度的测定和吸收光谱的扫描.

\section{3 结果与讨论}

3.1 碳点修饰多孔ZnO纳米棒的晶体结构、微观 形貌与元素分析

图1为系列碳点修饰多孔 $\mathrm{ZnO}$ 纳米棒光催化材 料的XRD图谱. 从曲线 $\mathrm{a}$ 中可知, 纯相 $\mathrm{ZnO}$ 纳米棒的 XRD 谱线的衍射峰对应标准锐钛矿结构 ZnO (JCPDS, No. 36-1451), 从图谱中可以明显观察到在 $2 \theta$ 为 $31.6^{\circ} 、 34.4^{\circ} 、 36.1^{\circ} 、 47.6^{\circ} 、 56.7^{\circ} 、 62.9^{\circ}$ 和 $68.0^{\circ}$ 的衍射峰分别对应 $\mathrm{ZnO}$ 的 $(100) 、(002)$ 、 (101)、(102)、(110)、(103)和(112)晶面. ${ }^{11,31}$ 图 $1(\mathrm{~b}-\mathrm{g})$ 为不同质量分数碳点修饰的多孔ZnO纳米棒 材料, 可以看出在所有碳点修饰 $\mathrm{ZnO}$ 样品中, 多孔 $\mathrm{ZnO}$ 纳米棒的晶面结构并未发生改变, 碳点相关峰 也因为碳点所占质量分数比较小 (小于 $5 \%$ ) 而未被 检测到. 系列样品XRD图谱说明, 经过不同质量分
数的碳点修饰, $\mathrm{ZnO}$ 的晶格结构并未受到干扰.

图2为纯相多孔ZnO纳米棒和 $\mathrm{CZn} 1.2$ 的扫描电 镜图, 其中, 图2 $\left(\mathrm{A}_{1}, \mathrm{~B}_{1}\right)$ 为低倍镜下的SEM图片, 图 $2\left(\mathrm{~A}_{2}, \mathrm{~B}_{2}\right)$ 为高倍镜下的SEM图片, 通过观察, 纯相多 孔 $\mathrm{ZnO}$ 棒生长比较均匀, 棒长为 $2-3 \mu \mathrm{m}$, 由粒径为 15-25 nm 的纳米颗粒组成. 由CZn1.2 (图2 $\left(\mathrm{B}_{1}, \mathrm{~B}_{2}\right)$ ) 扫 描电镜图可以看出经过碳点修饰后, 多孔ZnO纳米 棒结构未发生变化, 生长比较均匀, 其形貌与纯相 多孔ZnO纳米棒形貌一致.

由于用SEM无法直接观察到超小粒径的碳点, 因此通过HRTEM技术对该材料展开进一步的观察.

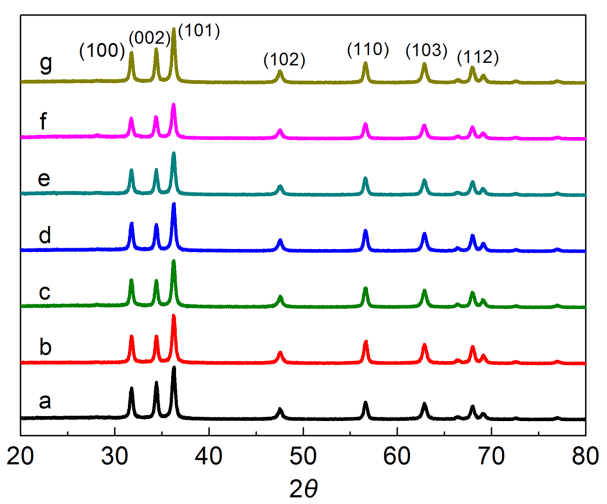

图1 系列样品的X射线衍射图

Fig.1 XRD patterns of the series samples

The mass fractions of $0 \%, 0.2 \%, 0.4 \%, 0.8 \%, 1.2 \%, 1.6 \%, 2.0 \% \mathrm{C}$-dots modified $\mathrm{ZnO}$ nanorods are denoted as (a) pure $\mathrm{ZnO} \mathrm{NR}$, (b) $\mathrm{CZn} 0.2$, (c) $\mathrm{CZn0.4,} \mathrm{(d)} \mathrm{CZn0.8,} \mathrm{(e)} \mathrm{CZn1.2,} \mathrm{(f)} \mathrm{CZn1.6}$; (g) CZn2.0, respectively.

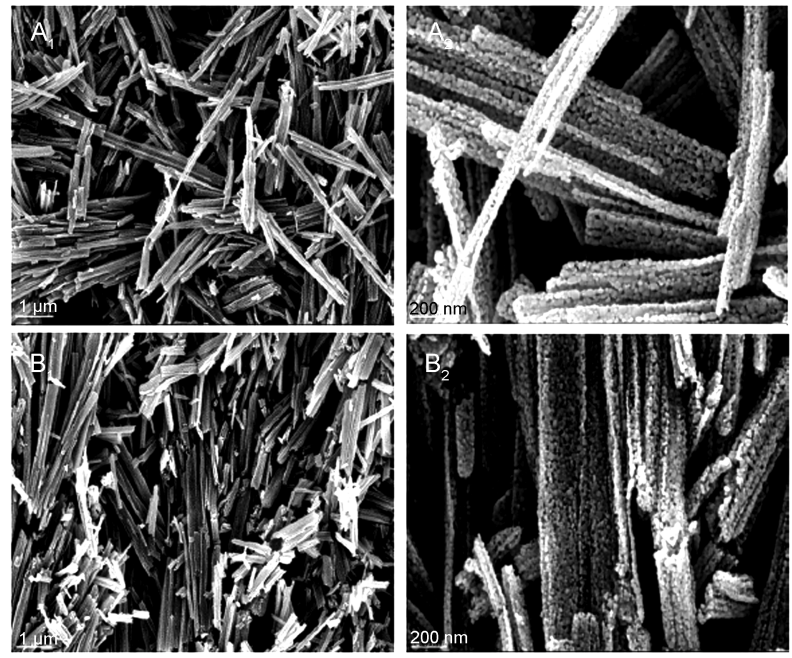

图2 $\left(\mathrm{A}_{1}, \mathrm{~A}_{2}\right)$ 纯相 $\mathrm{ZnO}$ 纳米棒和 $\left(\mathrm{B}_{1}, \mathrm{~B}_{2}\right) \mathrm{CZn1.2}$ 的SEM图片

Fig.2 SEM images of $\left(A_{1}, A_{2}\right)$ pure $\mathrm{ZnO} N R$ and $\left(B_{1}, B_{2}\right)$ CZn1.2

$\left(A_{1}, B_{1}\right)$ under low magnification; $\left(A_{2}, B_{2}\right)$ under high magnification 
图3为纯相多孔ZnO纳米棒和 $\mathrm{CZn} 1.2$ 的透射电镜图 片, 图 3 中 $\mathrm{A}_{1}$ 和 $\mathrm{B}_{1}$ 分别为纯相多孔 $\mathrm{ZnO}$ 和 $\mathrm{CZn} 1.2$ 低倍 镜下的透射电镜，对比两者并未发生明显的变化， 都是由 15-25 nm的纳米颗粒组成的多孔棒状结构.
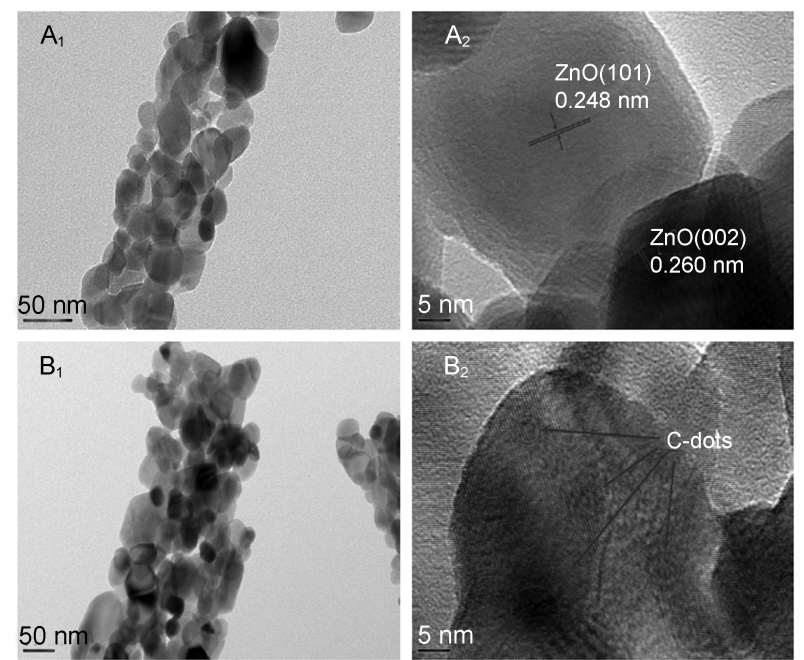

图3 $\left(\mathrm{A}_{1}, \mathrm{~A}_{2}\right)$ 纯相 $\mathrm{ZnO}$ 纳米棒和 $\left(\mathrm{B}_{1}, \mathrm{~B}_{2}\right) \mathrm{CZn1.2}$ 的 TEM图片

Fig.3 TEM images of $\left(A_{1}, A_{2}\right)$ pure $Z n O N R$ and $\left(B_{1}, B_{2}\right)$ CZn1.2

$\left(A_{1}, B_{1}\right)$ low resolution, $\left(A_{2}, B_{2}\right)$ high resolution
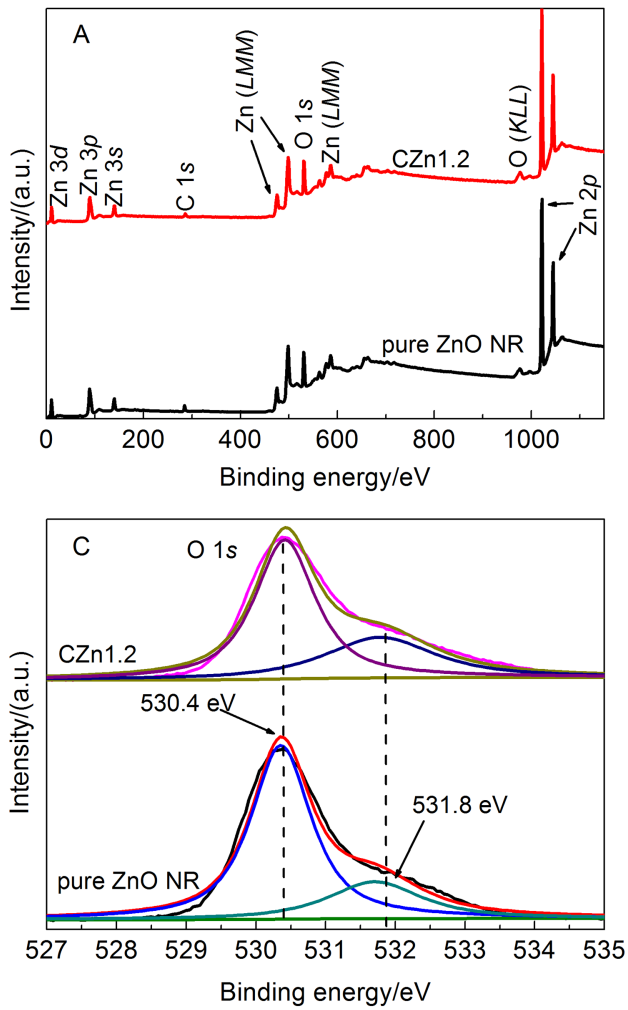

将两者进一步放大得到高分辨率的透射电镜图片 $\mathrm{A}_{2}$ 和 $\mathrm{B}_{2}$, 通过图片 $\mathrm{A}_{2}$ 可以观察到 $\mathrm{ZnO}$ 的(101)晶面和 (002)晶面对应XRD谱图中的衍射峰; 通过图片 $\mathrm{B}_{2}$ 除 了观察到 $\mathrm{ZnO}$ 的晶格结构外, 还能够观察到粒径为 3-5 $\mathrm{nm}$ 的量子点颗粒(红色圆圈区域)紧密接触 $\mathrm{ZnO}$ 纳米颗粒，由于在材料合成过程中除了利用碳 点对 $\mathrm{ZnO}$ 进行修饰之外, 并没有引入其他物质, 因此 这些量子点颗粒可在一定程度上被确定为表面修 饰的碳点. 需要通过XPS对合成的CZn1.2样品展开 进一步分析.

图4为纯相 $\mathrm{ZnO}$ 和 $\mathrm{CZn} 1.2$ 光催化剂的XPS元素 分析图, 图 $4 \mathrm{~A}$ 为纯相 $\mathrm{ZnO}$ 和 $\mathrm{CZn} 1.2$ 光催化剂的 XPS总谱分析, 可以观察到纯相 $\mathrm{ZnO}$ 和 $\mathrm{CZn} 1.2$ 样品 存在 $\mathrm{Zn} 、 \mathrm{O} 、 \mathrm{C}$ 三种元素. 进一步对纯相 $\mathrm{ZnO}$ 和

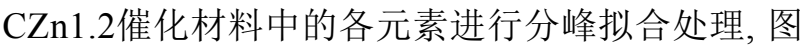
$4 \mathrm{~B}$ 为纯相 $\mathrm{ZnO}$ 和 $\mathrm{CZn} 1.2$ 光催化剂C $1 s$ 的特征峰, 相 比之下, 纯相 $\mathrm{ZnO}$ 和 $\mathrm{CZn} 1.2$ 光催化剂 C $1 s$ 的特征峰 有明显的不同，这是因为碳点成功修饰到多孔 $\mathrm{ZnO}$ 纳米棒表面, 使得 $\mathrm{CZn} 1.2$ 中 C $1 s$ 的XPS谱线发 生变化. 经过分峰处理得到285.2、287.2、289.2
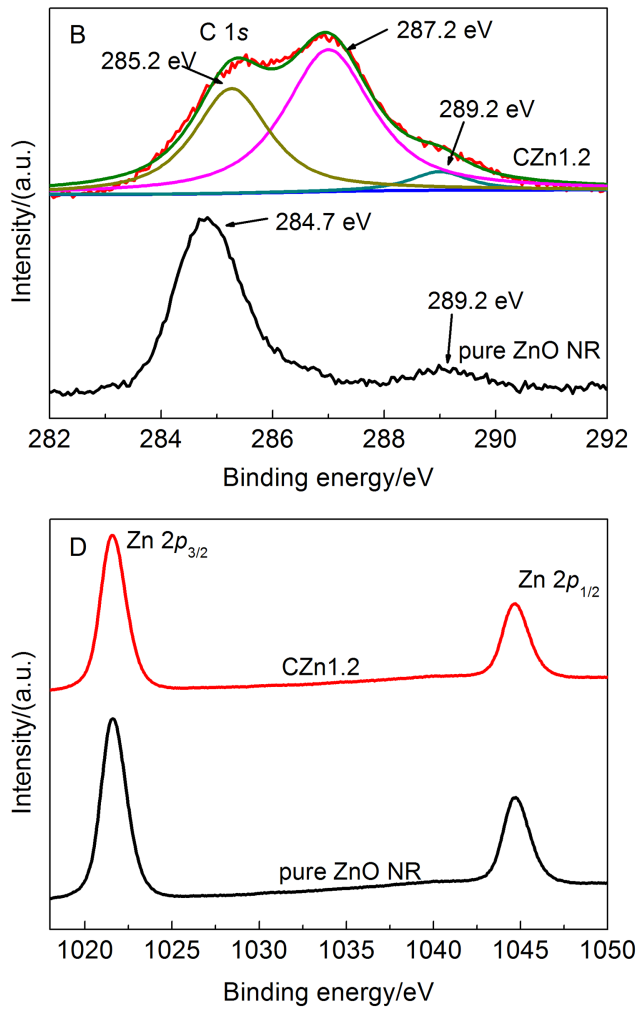

图4 纯相 ZnO和CZn1.2的XPS全谱图(A)和高分辨C $1 s(\mathrm{~B}), \mathrm{O} 1 \mathrm{~s}(\mathrm{C})$ 和 $\mathrm{Zn} 2 p(\mathrm{D})$ 的XPS谱图

Fig.4 (A) XPS survey spectra of pure ZnO NR and CZn1.2 (A) and high-resolution XPS spectra of $\mathrm{C} 1 s$ (B), O $1 s$ (C), and $\mathrm{Zn} 2 p$ (D) 
$\mathrm{eV}$ 三个特征峰，其中，285.2 $\mathrm{eV}$ 对应着 $s p^{2}$ 杂化的 $\mathrm{C}=\mathrm{C} ， 287.2$ 和 $289.2 \mathrm{eV}$ 对应着表面氧化基团 $\mathrm{C}=\mathrm{O}$ 和 $\mathrm{O}-\mathrm{C}=\mathrm{O},{ }^{12,21,32-34}$ 该结果表明碳点并不是以 还原态的形式存在，其表面存在大量的氧化集团. 图 $4 \mathrm{C}$ 为纯相 $\mathrm{ZnO}$ 和 CZn1.2的O $1 s$ 的特征峰, 可以发 现两种光催化材料的 $\mathrm{O} 1 s$ 的特征峰出现明显的不对 称情况, 通过分峰拟合进一步得到530.4和 531.8 $\mathrm{eV}$ 两个峰, 分别对应着纯相 $\mathrm{ZnO}$ 和 $\mathrm{CZn} 1.2$ 材料内部 的晶格氧与外部的化学吸附氧. ${ }^{35}$ 图 $4 \mathrm{D}$ 为纯相 $\mathrm{ZnO}$ 和 CZn1.2材料的 $\mathrm{Zn} 2 p_{3 / 2}$ 和 Zn $2 p_{1 / 2}$ 的特征峰, 分 别为 1022.0 和 $1045.2 \mathrm{eV},{ }^{36,37}$ 表明 $\mathrm{Zn}^{2+}$ 的存在. 通过 XRD、SEM、HRTEM以及XPS分析表明, 我们已 经成功合成了碳点修饰的多孔 $\mathrm{ZnO}$ 纳米棒样品, 并 且没有引入其他的杂质.

\section{2 碳点修饰多孔ZnO纳米棒光学吸收特性与光 催化性能}

图5为碳点修饰多孔ZnO纳米棒系列样品的紫 外-可见吸收光谱. 图 $5 \mathrm{a}$ 为纯相多孔 $\mathrm{ZnO}$ 纳米棒的紫 外-可见吸收光谱, 可以观察到纯相 $\mathrm{ZnO}$ 纳米棒的吸 收带边为 $380 \mathrm{~nm}$. 图5(b-g) 分别为添加不同质量分 数碳点修饰的 $\mathrm{ZnO}$ 纳米棒系列样品, 通过碳点的修 饰, 系列样品的吸收带边没有发生明显的变化, 这 可能是因为较少量的碳点修饰附着在 $\mathrm{ZnO}$ 纳米颗粒 的表面, 并未对 $\mathrm{ZnO}$ 的晶体结构造成影响. 通过观察 图5插图中纯相 $\mathrm{ZnO}$ 纳米棒与 $\mathrm{CZn} 1.2$ 的比较, 可以发 现 $\mathrm{CZn} 1.2$ 的颜色明显要比纯相 $\mathrm{ZnO}$ 纳米棒暗, 这是 因为经过碳点修饰的多孔ZnO棒竞争吸光使得颜色

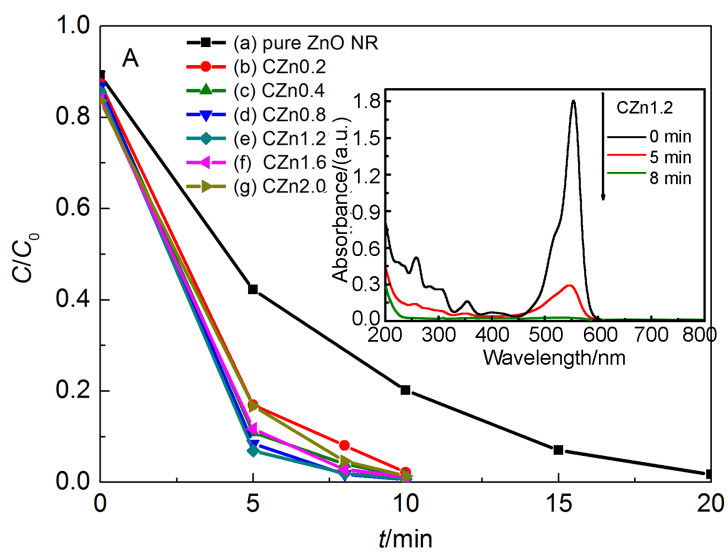

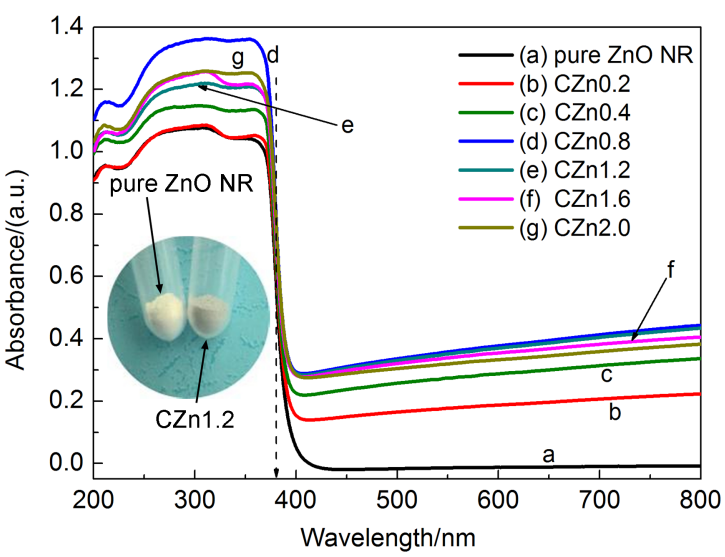

图5 系列样品的紫外-可见漫反射光谱

Fig.5 UV-Vis diffuse reflectance spectra of the series samples

较深, 因此, 经过碳点修饰的多孔ZnO棒在400-800 $\mathrm{nm}$ 波长范围内对光有了明显的吸收.

图6为氙灯光源照射下系列光催化剂对有机染 料 $\mathrm{RhB}\left(10 \mathrm{mg} \cdot \mathrm{L}^{-1}\right)$ 的光催化降解曲线和不同氙灯光 源条件下 CZn1.2对 RhB的降解曲线. 打开光源前, 将 光催化剂与染料 $R h B$ 溶液于暗态下搅拌 $30 \mathrm{~min}$ 达到 吸附平衡, 从图6A中发现, 纯 $\mathrm{ZnO}$ 纳米棒比修饰碳 点之后的系列样品対染料的吸附性能略弱, 这是因 为碳点也有一定的吸附能力. 从图 $6 \mathrm{~A}(\mathrm{a})$ 曲线可得, 在氙灯光源照射激发下，纯相 $\mathrm{ZnO}$ 纳米棒在 20 $\min$ 可实现对 $R h B$ 的完全脱色, 具有优异的光催化降 解性能; 经过修饰碳点后, CZn0.2 和 CZn2.0降解 $\mathrm{RhB}$ 的效率明显提高, $10 \mathrm{~min}$ 可实现对 $\mathrm{RhB}$ 的完全

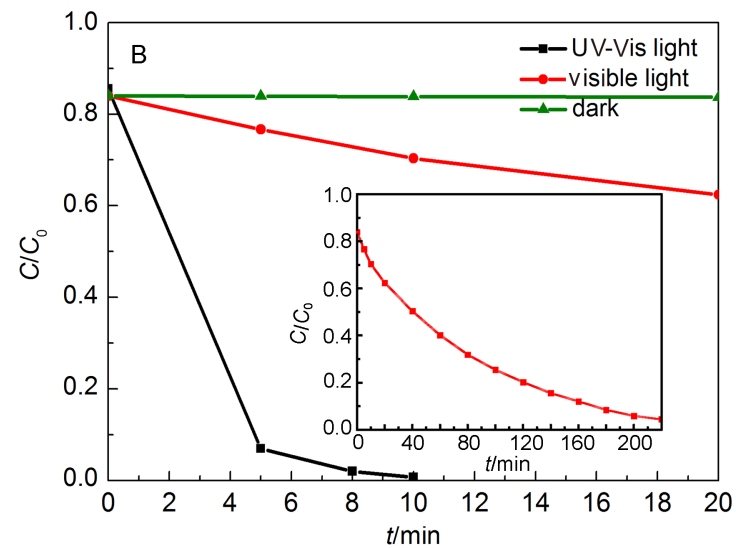

图6 (A)系列样品在氙灯光源照射下降解RhB的曲线图和(B)不同氙灯光源条件下CZn1.2降解RhB的曲线

Fig.6 (A) Photocatalysis degradation RhB curves of the series of samples under Xe-light irradiation; (B) photocatalysis degradation RhB curves of CZn1.2 under different Xe-light source conditions

Inset in figure A is UV-Vis absorption spectra of RhB irradiated by Xe-light irradiation for various durations in the presence of CZn1.2. Inset in figure $\mathrm{B}$ is the curve of $\mathrm{CZn} 1.2$ for photocatalysis degradation $\mathrm{RhB}$ under simulated visible light. 
脱色, 光催化性能为纯相 $\mathrm{ZnO}$ 纳米棒的 2 倍; $\mathrm{CZn} 0.4 、 \mathrm{CZn} 0.8 、 \mathrm{CZn} 1.2 、 \mathrm{CZn} 1.6$ (分别对应曲线 c、 d、e、f)系列样品均可在 $8 \mathrm{~min}$ 实现对 $R h B$ 的完 全脱色, 光催化性能为纯 $\mathrm{ZnO}$ 纳米棒的 2.5 倍, 通过 对比曲线c、d、e、f 可知, CZn1.2 (曲线e)具有最佳 光催化降解性能. 图6A中插图为氙灯照射下 $\mathrm{CZn} 1.2$ 降解RhB不同时间下的紫外-可见吸收光谱 图, 从图中可以看出在降解 $\mathrm{RhB}$ 过程中并无其他特 征峰的出现, 说明在其降解过程中并无其它中间产 物的产生. 图6B分别为氙灯光源照射、模拟可见光 和暗态下 CZn 1.2 对 RhB 的催化降解曲线, 在 10 $\min$ 时, 三种条件下CZn1.2对 RhB的降解率分别为 $99.4 \% 、 29.7 \%$ 和 $16.2 \%$. 图6B中插图为 $\mathrm{CZn} 1.2$ 在模 拟可见光照射下催化降解RhB的曲线, 可知在 220 $\min$ 时 RhB 降解比较完全, 这说明碳点光吸收作用和 上转换机制在氙灯光源照射催化降解过程中起到 微弱的作用. 系列光催化剂光催化降解染料的结果 表明, 经过碳点修饰可以明显提高多孔ZnO纳米棒 的光催化性能, 这主要是因为碳点在光催化体系中 起到转移光生电子受体的作用, ${ }^{21,38}$ 能够有效提高光 生电子与空穴的分离效率, 从而增强光催化降解性 能.

图7显示了不同自由基对催化活性的影响, 分 别添加 $2 \mathrm{mmol}$ 草酸钠 $(\mathrm{SO})$ 、正丁醇 $(\mathrm{N}-\mathrm{BA})$ 和异丙 醇(IPA)于 RhB溶液中作为光生空穴、光生电子和 羟基自由基的清除剂。通过图 7 可知，空白条件 (blank)下, 氙灯光源照射CZn1.2在 8 min可实现对

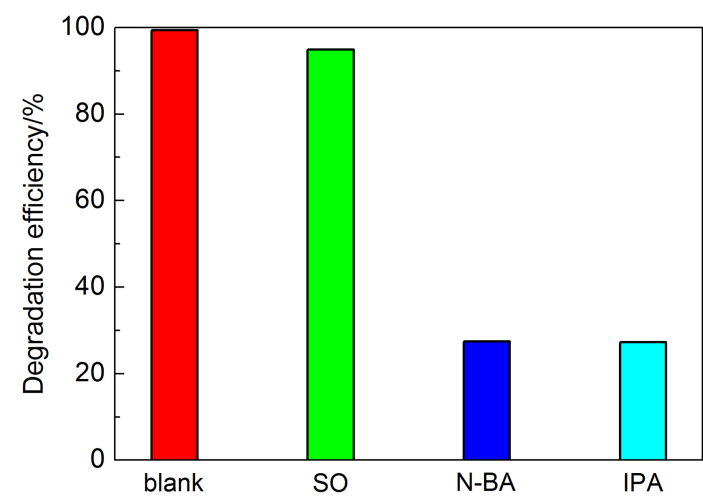

图7 添加不同自由基消除剂氙灯光源照射 8 min CZn1.2对 RhB降解的影响

Fig.7 Effects of different free-radical scavengers on degradation of RhB in the presence of CZn1.2 under Xelight irradiation for $8 \mathrm{~min}$

SO: soduim oxalate; N-BA: $n$-butyl alcohol; IPA: isopropyl alcohol
$\mathrm{RhB} 99.4 \%$ 的降解, 添加 $2 \mathrm{mmol}$ 草酸钠后, CZn1.2在 $8 \mathrm{~min}$ 可实现对 RhB 94.9\%的降解, 说明空穴不是决 定光催化降解过程中的主要活性物种. 然而分别添 加 $2 \mathrm{mmol}$ 正丁醇和异丙醇后，氙灯光源照射下 $\mathrm{CZn} 1.2$ 在 $8 \mathrm{~min}$ 可分别实现对 $\mathrm{RhB} 27.3 \%$ 和 $27.4 \%$ 的 降解, 这说明, 通过光生电子产生的羟基自由基是 影响CZn1.2光催化性能的主要活性物种. 而分别清 除光生电子和羟基自由基时, $\mathrm{CZn} 1.2$ 仍可实现对 RhB 27.0\%左右的降解率, 说明空穴在降解过程中 起到一定作用. 通过分析, 在氙灯光源照射下碳点 修饰 $\mathrm{ZnO}$ 的光催化降解过程中, 光生空穴起到一定 作用, 但并非主要活性物种, 而在导带处由光生电 子产生的羟基自由基起到主要作用.

图8为氙灯光源照射下系列光催化剂对有机污 染物苯酚 $\left(20 \mathrm{mg} \cdot \mathrm{L}^{-1}\right)$ 的光催化降解曲线. 打开光源 前, 同样将光催化剂与苯酚溶液于暗态下搅拌 30 $\min$ 达到吸附平衡. 打开光源后, 每隔 $15 \mathrm{~min}$ 取一次 降解溶液进行分析，图8A中显示氙灯光源照射 60 $\min$ 系列样品降解苯酚的曲线, 从图 $8 \mathrm{~A}$ 中可得, 在氙 灯光源照射激发下, 经过 $60 \mathrm{~min}$, 纯相 $\mathrm{ZnO}$ 纳米棒 (图 $8 \mathrm{~A}(\mathrm{a})$ )对苯酚的降解率为 $86.4 \%$, 而经过碳点修 饰的多孔ZnO纳米棒光催化降解苯酚的性能有明显 的提高, 经过 $60 \mathrm{~min}, \mathrm{CZn} 0.4 、 \mathrm{CZn} 0.8 、 \mathrm{CZn} 1.2$ 、 CZn1.6、CZn2.0 (分别对应图8A(b, c, d, e, f))系列 样品对苯酚的降解率分别为 $93.6 \%$ 、93.9\%、 $94.3 \% 、 93.5 \% 、 93.3 \%$. 为描述系列光催化剂降解 苯酚中间产物的变化, 如图8(a-f)所示, 分别对应在 不同光催化剂存在情况下, 不同时间的降解溶液的 紫外吸收光谱的扫描曲线, 其中P代表苯酚, $\mathrm{H}$ 代表 氢醌. 通过观察扫描图谱, 降解过程中除了出现苯 酚的特征峰(210.0和 $269.5 \mathrm{~nm})$ 之外, 同时出现了中 间产物氢醌的特征峰 $(291 \mathrm{~nm})$, 无其他产物特征峰 的出现, 说明经过碳点修饰的 $\mathrm{ZnO}$ 纳米棒对苯酚的 光催化降解性能有进一步的提高, 并且, 系列光催 化剂在光催化降解苯酚过程中, 出现了中间产物, 需要进一步分析光催化降解苯酚的过程.

系列光催化剂降解苯酚的过程如图9所示. 经 过分析, 纯 $\mathrm{ZnO}$ 纳米棒和碳点修饰多孔 $\mathrm{ZnO}$ 纳米棒 在氙灯光源照射下光催化降解苯酚主要存在两个 过程: (I) 是苯酚被氧化直接通过开环反应, 形成最 终产物 $\mathrm{CO}_{2}$ 和 $\mathrm{H}_{2} \mathrm{O}$, 此过程需要 $28 \mathrm{e}$ 参与反应并伴随 着 $7 \mathrm{O}_{2}$ 的消耗; (II) 是苯酚被氧化经过中间产物氢醌, 

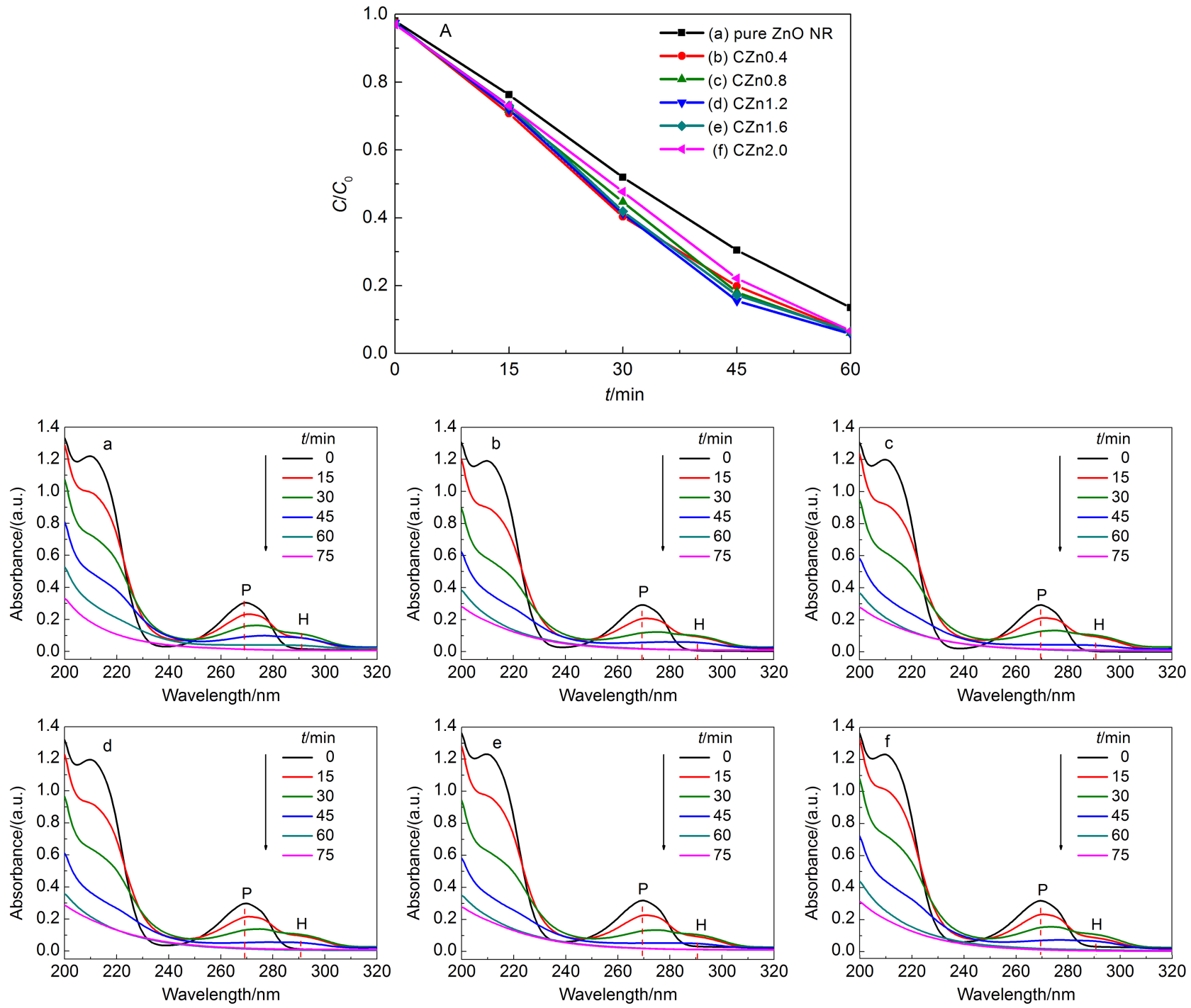

图8 (A)不同样品对苯酚的降解效果曲线; (a-f)氙灯光源照射下不同样品在不同时间下降解苯酚的紫外吸收光谱图

Fig.8 (A) Effect of different samples on phenol degradation; (a-f) UV absorption spectra of phenol irradiated by Xe-light irradiation for various durations in the presence of different samples

(a) pure ZnO NR; (b) $\mathrm{CZn0.4;} \mathrm{(c)} \mathrm{CZn0.8;} \mathrm{(d)} \mathrm{CZn1.2;} \mathrm{(e)} \mathrm{CZn1.6;} \mathrm{(f)} \mathrm{CZn2.0.} \mathrm{P} \mathrm{and} \mathrm{H} \mathrm{indicate} \mathrm{phenol} \mathrm{and} \mathrm{hydroquinone,} \mathrm{respectively.}$

然后开环形成最终产物 $\mathrm{CO}_{2}$ 和 $\mathrm{H}_{2} \mathrm{O}$, 此过程首先需要

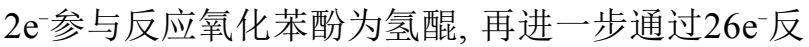
应发生开环反应被氧化为最终产物, 降解苯酚的活 性基团主要为催化剂表面产生的・OH. ${ }^{39}$ 由图8和图 9 可知, 光催化降解苯酚过程中, 苯酚的浓度在不断 下降, 说明苯酚不断地减少, 而中间产物氢醌的浓 度呈现先上升后下降的过程, 在 $30 \mathrm{~min}$ 达到最大值, 这是因为在前 $30 \mathrm{~min}$, 苯酚的浓度较高, 在光催化过 程中除了自身发生直接开环反应外, 同时部分转化 为中间产物氢醌, 随着 $30 \mathrm{~min}$ 后苯酚的浓度降低, 转 化为氢醌的速率逐渐变慢, 中间产物氢醌的浓度开 始降低, 最终在氙灯光源照射下实现光催化剂对苯
酚的完全降解矿化为 $\mathrm{CO}_{2}$ 和 $\mathrm{H}_{2} \mathrm{O}$.

\section{3 碳点修饰多孔ZnO纳米棒光催化机理分析}

图10为碳点修饰多孔ZnO纳米棒光催化降解有 机污染物的机理分析. 在氙灯光源照射激发下, $\mathrm{ZnO}$ 半导体价带处的电子吸收光子获得能量被激发 到导带处形成光生电子, 相对应的在 $\mathrm{ZnO}$ 价带处形 成光生空穴, 并分别转移到半导体表面. 经过碳点 修饰后, 碳点与 $\mathrm{ZnO}$ 紧密接触并作为光生电子的载 体, 能够促进光生电子与空穴的有效分离, 增强光 催化性能, 光生电子转移到碳点并在碳点表面催化 表面吸附氧形成 $\mathrm{O}_{2}^{-}, \mathrm{O}_{2}^{-} \cdot$ 进一步转化形成・ $\mathrm{OH}$, 和 光生空穴, 共同催化氧化 RhB和苯酚, 达到光催化降 


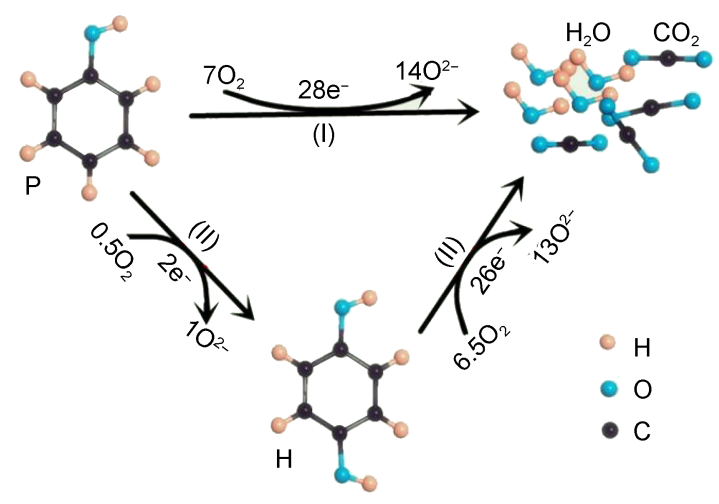

图9 苯酚的光催化降解途径分析图

Fig.9 Proposed photodegradation pathways of phenol

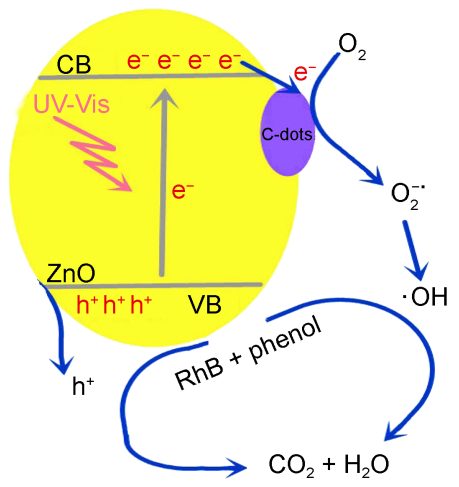

图10 碳点修饰 $\mathrm{ZnO}$ 纳米棒降解 $\mathrm{RhB}$ 和苯酚的光催化 机理图

Fig.10 Photocatalytic mechanism of C-dots modified $\mathrm{ZnO}$ NR photocatalyst for RhB and phenol degradation

VB: valence band; $\mathrm{CB}$ : conduction band

解有机污染物的效果, 并最终生成 $\mathrm{CO}_{2}$ 和 $\mathrm{H}_{2} \mathrm{O}^{40}$

\section{4 结 论}

本实验成功制备碳点修饰多孔ZnO纳米棒系列 光催化剂, 并对其进行光催化性能研究. 经过碳点 修饰, 多孔ZnO纳米棒光催化降解RhB性能明显提 高, 其中 CZn1.2的光催化性能为纯 $\mathrm{ZnO}$ 纳米棒的 2.5 倍, 光催化降解苯酚的性能也有一定的提升, 通 过检测活性自由基对光催化性能的影响, 发现在光 催化降解过程中起主要作用的是・ $\mathrm{OH}$, 而碳点在光 催化剂中起到光生电子载体的作用, 能够促进光生 电子与空穴的有效分离, 从而增强光催化性能.

\section{References}

(1) Maeda, K.; Teramura, K.; Lu, D.; Takata, T.; Saito, N.; Inoue, Y.; Domen, K. Nature 2006, 440, 295. doi: 10.1038/440295a
(2) Bu, Y. Y.; Chen, Z. Y. RSC Adv. 2014, 4, 45397. doi: 10.1039/C4RA06641C

(3) Kudo, A.; Miseki, Y. Chem. Soc. Rev. 2009, 38, 253. doi: 10.1039/B800489G

(4) Xie, J.; Wang, H.; Duan, M. Acta Phys. -Chim. Sin. 2011, 27, 193. [谢 娟, 王 虎, 段 明. 物理化学学报, 2011, 27, 193.] doi: 10.3866/PKU.WHXB20110124

(5) Li, D. H.; Yang, D. J.; Quan, F. Y.; Wang, B. B.; Zhang, L. J.; Zhu, S. S.; Wang, L. J. Nano Reports 2015, 1, 29.

(6) Liu, H.; Wu, X. F.; Li, X. Q.; Wang, J.; Fan, X. M. Chin. J. Catal. 2014, 35, 1997. [刘红, 鸟小凤, 李湘奇, 王 婕, 范希 梅. 催化学报, 2014, 35, 1997.] doi: 10.1016/S18722067(14)60198-4

(7) Xu, F.; Sun, L. Energy Environ. Sci. 2011, 4, 818. doi: 10.1039/C0EE00448K

(8) Huang, J.; Yin, Z. G.; Zheng, Q. D. Energy Environ. Sci. 2011, 4, 3861. doi: 10.1039/c1ee01873f

(9) Shen, G.; Cho, J. H.; Yoo, J. K.; Yi, G.; Lee, C. J. J. Phys. Chem. $B$ 2005, 109, 5491. doi: 10.1021/jp045237m

(10) Li, X. Q.; Fan, Q. F.; Li, G. L.; Huang, Y. H.; Gao, Z.; Fan, X. M.; Zhang, C. L.; Zhou, Z. W. Acta Phys. -Chim. Sin. 2015, 31, 783. [李湘奇, 范庆飞, 李广立, 黄瑶翰, 高 照, 范希梅, 张朝 良, 周祚万. 物理化学学报, 2015, 31, 783.] doi: 10.3866/PKU.WHXB201502062

(11) Zhu, C. Q.; Lu, B. G.; Su, Q.; Xie, E. Q.; Lan, W. Nanoscale 2012, 4, 3060. doi: 10.1039/c2nr12010k

(12) Samadi, M.; Shivaee, H. A.; Zanetti, M.; Pourjavadi, A.; Moshfegh, A. J. Mol. Catal. A: Chem. 2012, 359, 42. doi: 10.1016/j.molcata.2012.03.019

(13) Bu, Y. Y.; Chen, Z. Y. J. Power Sources 2014, 272, 647. doi: 10.1016/j.jpowsour.2014.08.127

(14) Sun, X.; Li, Q.; Jiang, J. C.; Mao, Y. B. Nanoscale 2014, 6, 8769. doi: 10.1039/C4NR01146E

(15) Sin, J. C.; Lam, S. M.; Satoshi, I.; Lee, K. T.; Mohamed, A. R. Appl. Catal. B 2014, 148-149, 258.

(16) Wang, X. W.; Yin, L. C.; Liu, G.; Wang, L. Z.; Saito, R.; Lu, G. Q.; Cheng, H. M. Energy Environ. Sci. 2011, 4, 3976. doi: 10.1039/c0ee00723d

(17) Zhang, Y.; Lin, S.; Zhang, Y.; Song, X. M. Acta Phys. -Chim. Sin. 2013, 29, 2399. [张 宇, 林 申, 张 钰, 宋溪明. 物理化 学学报, 2013, 29, 2399.] doi: 10.3866/PKU.WHXB201309061

(18) Wu, L. L.; Tian, R. X.; Zhao, Q.; Chang, Q.; Hu, S. L. Chem. J. Chin. Univ. 2014, 35, 717. [武玲玲, 田瑞雪, 赵 清, 常 青, 胡 胜亮. 高等学校化学学报, 2014, 35, 717.]

(19) Sun, X.; Tuo, J. Q.; Yang, W. Y.; Yang, D. J. Nano Reports 2015, 2,51

(20) Li, H.; He, X.; Kang, Z.; Huang, H.; Liu, Y.; Liu, J.; Lian, S.; Tsang, C. H.; Yang, X.; Lee, S. T. Angew. Chem. Int. Edit. 2010, 49, 4430. doi: 10.1002/anie.200906154

(21) Ming, H.; Ma, Z.; Liu, Y.; Pan, K. M.; Yu, H.; Wang, F.; Kang, Z. H. Dalton Trans. 2012, 41, 9526. doi: 10.1039/c2dt30985h 
(22) De, B.; Voit, B.; Karak, N. RSC Adv. 2014, 102, 58453.

(23) Zhang, H. C.; Huang, H.; Ming, H.; Li, H. T.; Zhang, L. L.; Liu, Y.; Kang, Z. H. J. Mater. Chem. 2012, 22, 10501. doi: $10.1039 / \mathrm{c} 2 \mathrm{jm} 30703 \mathrm{k}$

(24) Li, H. T.; Kang, Z. H.; Liu, Y.; Lee, S. T. J. Mater. Chem. 2012, 22, 24230. doi: 10.1039/c2jm34690g

(25) Wang, J.; Huang, H. M.; Xu, Z. Z.; Kou, J. H.; Lu, C. H. Curr. Org. Chem. 2014, 18, 1346. doi: $10.2174 / 1385272819666140424214022$

(26) Yu, W. L.; Zhang, J. F.; Peng, T. Y. Appl. Catal. B 2016, 181, 220. doi: 10.1016/j.apcatb.2015.07.031

(27) Jassby, D.; Farner, B. J.; Wiesner, M. Environ. Sci. Technol. 2012, 46, 6934. doi: 10.1021/es202009h

(28) Skompska, M.; Zarębska, K. Electrochim. Acta 2014, 127, 467. doi: 10.1016/j.electacta.2014.02.049

(29) Liu, J.; Liu, Y.; Liu, N. Y.; Han,Y. Z.; Zhang, X.; Huang, H.; Lifshitz, Y.; Lee, S. T.; Zhong, J.; Kang, Z. H. Science 2015, 347, 970. doi: 10.1126/science.aaa3145

(30) Peng, Y.; Qin, S.; Wang, W. S.; Xu, A. W. CrystEngComm 2013, 15, 6518. doi: 10.1039/c3ce40798e

(31) Xu, Y. G.; Xu, H.; Li, H. M.; Xia, J. X.; Liu, C. T.; Liu, L. J. Alloy. Compd. 2011, 509, 3286. doi: 10.1016/j.jallcom.
2010.11 .193

(32) Zhang, H.; Fan, X.; Quan, X.; Chen, S.; Yu, H. Environ. Sci. Technol. 2011, 45, 5731. doi: 10.1021/es2002919

(33) Wen, Y.; Ding, H.; Shan, Y. Nanoscale 2011, 3, 4411. doi: $10.1039 / \mathrm{c} 1 \mathrm{nr} 10604 \mathrm{j}$

(34) Dong, Y. Q.; Wang, R. X.; Li, H.; Shao, J. W.; Chi, Y. W.; Lin, X. M.; Chen, G. N. Carbon 2012, 50, 2810. doi: 10.1016/j.carbon.2012.02.046

(35) Peng, W. Q.; Qu, S. C.; Cong, G. W.; Wang, Z. G. Cryst. Growth Des. 2006, 6, 1518. doi: 10.1021/cg0505261

(36) Li, Y.; Zhang, B. P.; Zhao, J. X. J. Alloy. Compd. 2014, 586, 663. doi: 10.1016/j.jallcom.2013.10.085

(37) Rajbongshi, B. M.; Samdarshi, S. K. Appl. Catal. B 2014, 144, 435. doi: 10.1016/j.apcatb.2013.07.048

(38) Yu, X. J.; Liu, J. J.; Yu, Y. C.; Zuo, S. L.; Liu, B. S. Carbon 2014, 68, 718. doi: 10.1016/j.carbon.2013.11.053

(39) Su, R.; Tiruvalam, R.; He, Q.; Dimitratos, N.; Kesavan, L.; Hammond, C.; Lopez-Sanchez, J. A.; Bechstein, R.; Kiely, C. J.; Hutchings, G. J.; Besenbacher, F. ACS Nano 2012, 6, 6284. doi: $10.1021 / \mathrm{nn} 301718 \mathrm{v}$

(40) Xue, C.; Wang, T.; Yang, G. D.; Yang, B. L.; Ding, S. J. J. Mater. Chem. A 2014, 2, 7674. doi: 10.1039/c4ta01190b 